CA. The youngest patients had the most severe lissencephaly but no correlation was found between the severity of the malformation as graded by MRI and the severity of clinical disease. One child age $21 / 2$ with frontal lobes most severely affected was walking, speaking in 3 to 4 word sentences, and following instructions appropriate to her age, and physical examination revealed only very mild hypotonia. The anatomical deformity in the brain was not associated with any delay in myelination in patients in this study. Seizures occurred in all but one of the patients greater than four months of age; they began with infantile spasms or focal motor seizures and tonic seizures commonly occurred by the end of the second year. Hypsarrhythmia was present in two patients (Barkovich AJ et al. The spectrum of lissencephaly: report of ten patients analyzed by magnetic resonance imaging. Ann Neurol August 1991; $\underline{30}: 139-146)$.

COMMENT. The normal pattern and rate of myelination and the absence of porencephalies or other destructive lesions in 9 of the 10 patients in this report suggests that the arrest of neuronal migration in lissencephaly results from the absence of molecular components of the glial fibers or intracellular matrix. Lissencephaly is described in two types according to clinical, radiological, and pathological characteristics. In type I lissencephaly, agyria and pachygyria regions have a molecular layer, an outer cellular layer, a cell-sparse layer, and a deep cellular layer composed of heterotopic incompletely migrated neurons. In type II lissencephaly, the thickened cortex is disorganized without evidence of layers, and hydrocephalus and multiple anomalies are usually present.

\title{
GENERALIZED CORTICAL DYSPLASIA
}

The clinical and neuropathological findings in three children with diffuse cortical dysplasia are reported from the Departments of Pathology, Neurology and Pediatrics, University of Rochester Medical Center, Rochester, NY. The children had seizures unresponsive to antiepileptic medication throughout life, they were profoundly mentally retarded, and they died after a progressive neurological deterioration at ages 10 months, 3 years and 7 years. Pathologically, the brains showed generalized cortical dysplasia, without any major malformation of the external gyral pattern. The cortical gray matter was thickened and the demarcation of the gray-white matter junction was indistinct. Microscopically, the cortex contained increased numbers of large neurons with disordered cortical lamination and heterotopic neurons scattered throughout the white matter. The cortical involvement was multifocal involving all lobes of the cerebral hemispheres, and the underlying myelin was pale and rarified. (Kazee AM et al. Generalized cortical dysplasia. Clinical and pathologic aspects. Arch Neurol August 1991; 48:850-853.

COMMENT. The authors report these cases as a newly recognized entity of abnormal neuronal migration. In contrast to classic lissencephaly no distinct lamination of the cortical layers was present 
and the underlying myelin was also involved. The cause of Generalized Cortical Dysplasia is unknown but a genetic etiologic factor is suspected.

\section{HEAD SIZE AND COGNITION IN VERY LOW BIRTH WEIGHT (VLBW) INFANTS}

The effect of VLBW $(<1.5 \mathrm{~kg})$ and subnormal head size at 8 months of age (corrected for prematurity) on neurocognitive abilities at school age was investigated by a longitudinal follow up of 249 children born between 1977 to 1979 and admitted to the neonatal intensive care unit at Rainbow Babies and Children's Hospital, Cleveland, $\mathrm{OH}$. Head size was subnormal at birth in $12 \%$, at term $23 \%$, and at 8 months $13 \%$. As compared with 216 children with normal head sizes, the 33 children with subnormal head sizes at the age of 8 months had significantly lower mean birth weights and higher neonatal risk scores and at 8 years of age had a higher incidence of neurologic impairment and lower IQ scores. A subnormal head size at 8 months of age was predictive of poor verbal and performance IQ scores at 8 years of age; lower scores for receptive language, speech, reading, mathematics and spelling; and a higher incidence of hyperactivity. (Hack M et al. Effect of very low birth weight and subnormal head size on cognitive abilities at school age. N Engl J Med July 25, 1991; $\underline{325: 231-237)}$

COMMENT. A relation between subnormal head circumference and cognitive functioning has been reported in many different studies, including children with learning disabilities. Improvements in neonatal growth and development may depend on nutrition of chronically ill neonates and environmental enrichment after discharge from hospital. This study shows that the determination of the head circumference at 8 months of age is a useful means of predicting subsequent cognitive deficits and difficulties in school. Volpe JJ, in an editorial (N Engl J Med 1991; 325:276-277), stresses the need for a better method of detection of brain injury in VLBW infants since $77 \%$ of those with normal head circumference at 8 months had IQs below 85 and an academic skill index below 80 in the study by Hack et al. Those infants with a small head circumference at 8 months of age made up only a minority of infants who subsequently had cognitive deficits. Cranial ultrasonography detects overt brain lesions but not the subtle lesions in the periventricular white matter. MRI may detect periventricular injury missed by ultrasound and CT scanning; it is also used to measure the progress and degree of myelination in the human infant in the first months of life. A decreased rate of brain growth in the first months of life might identify a larger proportion of at risk infants and justify further study with the MRI in selected patients.

Collin MF and associates from the Department of Pediatrics, Loyola University Medical Center, Maywood, IL report that normal infant development is poorly predictive of continued normal development in the extremely low birth weight infant (Pediatrics July 